Delft University of Technology

\title{
On-chip integration of Si/SiGe-based quantum dots and switched-capacitor circuits
}

Xu, Y.; Unseld, F. K.; Corna, A.; Zwerver, A. M.J.; Sammak, A.; Brousse, D.; Samkharadze, N.; Amitonov, S. V.; Veldhorst, M.; Scappucci, G.

DOI

10.1063/5.0012883

Publication date

2020

Document Version

Final published version

Published in

Applied Physics Letters

\section{Citation (APA)}

Xu, Y., Unseld, F. K., Corna, A., Zwerver, A. M. J., Sammak, A., Brousse, D., Samkharadze, N., Amitonov, S. V., Veldhorst, M., Scappucci, G., Ishihara, R., \& Vandersypen, L. M. K. (2020). On-chip integration of Si/SiGe-based quantum dots and switched-capacitor circuits. Applied Physics Letters, 117(14), [144002]. https://doi.org/10.1063/5.0012883

Important note

To cite this publication, please use the final published version (if applicable).

Please check the document version above.

\section{Copyright}

Other than for strictly personal use, it is not permitted to download, forward or distribute the text or part of it, without the consent of the author(s) and/or copyright holder(s), unless the work is under an open content license such as Creative Commons.

\section{Takedown policy}

Please contact us and provide details if you believe this document breaches copyrights.

We will remove access to the work immediately and investigate your claim. 
Green Open Access added to TU Delft Institutional Repository

'You share, we take care!' - Taverne project

https://www.openaccess.nl/en/you-share-we-take-care

Otherwise as indicated in the copyright section: the publisher is the copyright holder of this work and the author uses the Dutch legislation to make this work public. 


\section{On-chip integration of Si/SiGe-based quantum dots and switched-capacitor circuits}

Cite as: Appl. Phys. Lett. 117, 144002 (2020); https://doi.org/10.1063/5.0012883

Submitted: 06 May 2020. Accepted: 22 August 2020 . Published Online: 06 October 2020

Y. Xu (D), F. K. Unseld (D), A. Corna (D), A. M. J. Zwerver (D), A. Sammak (D), D. Brousse (iD, N. Samkharadze (D), S. v. Amitonov (D), M. Veldhorst (D), G. Scappucci (D), R. Ishihara (D), and L. M. K. Vandersypen (D)

\section{COLLECTIONS}

This paper was selected as Featured
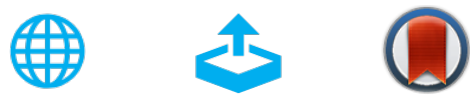

\section{ARTICLES YOU MAY BE INTERESTED IN}

Experimental investigation of amplification, via a mechanical delay-line, in a rainbow-based metamaterial for energy harvesting

Applied Physics Letters 117, 143902 (2020); https://doi.org/10.1063/5.0023544

Synthetic chiral magnets promoted by the Dzyaloshinskii-Moriya interaction Applied Physics Letters 117, 130503 (2020); https://doi.org/10.1063/5.0021184

Reduction of charge noise in shallow GaAs/AIGaAs heterostructures with insulated gates Applied Physics Letters 117, 133504 (2020); https://doi.org/10.1063/5.0026259






\title{
On-chip integration of Si/SiGe-based quantum dots and switched-capacitor circuits $\odot$
}

\author{
Cite as: Appl. Phys. Lett. 117, 144002 (2020); doi: 10.1063/5.0012883 \\ Submitted: 6 May 2020 - Accepted: 22 August 2020 . \\ Published Online: 6 October 2020
}



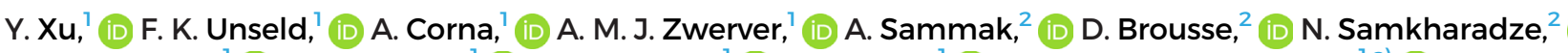
S. V. Amitonov,' (D) M. Veldhorst, ' (D) G. Scappucci,' (D) R. Ishihara,' (D) and L. M. K. Vandersypen ${ }^{1, a)}$ (D)

\author{
AFFILIATIONS \\ ${ }^{7}$ QuTech and Kavli Institute of Nanoscience, Delft University of Technology, Lorentzweg 1, 2628 CJ Delft, The Netherlands \\ ${ }^{2}$ QuTech and Netherlands Organization for Applied Scientific Research (TNO), Stieltjesweg 1, 2628 CK Delft, The Netherlands \\ ${ }^{\text {a) }}$ Author to whom correspondence should be addressed: I.m.k.vandersypen@tudelft.nl
}

\begin{abstract}
Solid-state qubits integrated on semiconductor substrates currently require at least one wire from every qubit to the control electronics, leading to a so-called wiring bottleneck for scaling. Demultiplexing via on-chip circuitry offers an effective strategy to overcome this bottleneck. In the case of gate-defined quantum dot arrays, specific static voltages need to be applied to many gates simultaneously to realize electron confinement. When a charge-locking structure is placed between the quantum device and the demultiplexer, the voltage can be maintained locally. In this study, we implement a switched-capacitor circuit for charge-locking and use it to float the plunger gate of a single quantum dot. Parallel plate capacitors, transistors, and quantum dot devices are monolithically fabricated on a Si/SiGe-based substrate to avoid complex off-chip routing. We experimentally study the effects of the capacitor and transistor size on the voltage accuracy of the floating node. Furthermore, we demonstrate that the electrochemical potential of the quantum dot can follow a $100 \mathrm{~Hz}$ pulse signal while the dot is partially floating, which is essential for applying this strategy in qubit experiments.
\end{abstract}

Published under license by AIP Publishing. https://doi.org/10.1063/5.0012883

Silicon spin qubits based on gate-defined quantum dots have recently been realized on $\mathrm{Si} / \mathrm{SiGe}$ substrates with high fidelity. ${ }^{1-6}$ Given their compatibility with current semiconductor fabrication techniques and potential for easy integration with classical electronics, these quantum dots are considered to be a promising basis for quantum computers. ${ }^{7-10}$ Nevertheless, a fault-tolerant quantum computer requires millions of qubits. ${ }^{11}$ Even if quantum dots are designed to be identical, the required gate bias voltage still differs among the dots due to non-uniformities in the substrate and variations during the fabrication process. For every dot in a typical quantum dot array, one gate controls the electrochemical potential and a second controls the tunnel barrier to the next dot in the array. Each gate is connected to a separate room temperature digital-to-analog converter (DAC) through the bond wires from the chip to the sample carrier and the dilution refrigerator wiring. However, this linear approach clearly poses a bottleneck to scaling up the number of qubits. By comparison, today's classical processor chips have only about 2000 contact pins, while billions of transistors can be integrated and operated on a single chip. This large ratio between active components and pins is described by Rent's rule and is made possible by implementing shared control methods. ${ }^{12}$ In order to operate the millions of qubits for practical quantum computation, similar methods will, therefore, have to be implemented in quantum integrated circuits.

Inspired by the classical dynamic random-access memory (DRAM) matrix that uses word lines and bit lines to address a large number of storage cells, ${ }^{13}$ proposals for controlling spin qubits using word and bit lines exist. ${ }^{14-18}$ Another concept that can be borrowed from DRAM is charge-locking, which, when combined with demultiplexers, allows us to significantly reduce the number lines going off-chip. $^{15,16,19,20}$ In DRAM, the stored voltage encodes "0" or " 1 ," according to a threshold. In contrast, the voltage maintained on a quantum dot gate needs to be a precise analog value. The required precision of such a stored voltage ranges from $1 \mu \mathrm{V}$ to $1 \mathrm{mV}$, depending on the gate function and coupling of the gate to the dot potential. ${ }^{15,18}$ Charge-locking is thus used in the form of a sample-and-hold circuit; when the input line is electrically detached, the gate of the quantum dot is floating and the voltage maintains there for a certain period. Although the primary role for DC gates of quantum dots is to achieve electron confinement, additional voltage pulses must be applied to these gates for qubit experiments. For example, in a commonly used single-shot readout method to determine the state of an electron spin, a few $\mathrm{kHz}$ signal is applied to the gate to load, read, and empty a 
quantum dot. ${ }^{21,22}$ When a switched-capacitor (SC) circuit is integrated with these gates as an interface, the extra transistor or capacitor should not affect the voltage pulses arriving at the quantum dot gates. Prototypes have been made with on-chip or off-chip integrated floating gate circuits and GaAs quantum dots. ${ }^{19,20}$ For silicon-based quantum dots, switching circuits have been integrated with quantum devices on-chip. ${ }^{23,24}$ In addition, charge-storage devices and quantum devices have been fabricated using the same CMOS process and connected through wire bonds. ${ }^{25,26}$ However, in silicon, a fully on-chip integrated solution, without the need for wire bonds, is still waiting to be achieved.

In this study, we integrate a switched-capacitor circuit containing an n-type transistor and a holding capacitor with a single quantum dot on a Si/SiGe-based substrate. We analyze the parameters that affect the variability of the floating gate voltage and experimentally study the impact of the size of the capacitor and the transistor. In addition, we apply a pulsed voltage to one of the quantum dot gates while floating another gate, as a relevant test for qubit measurements.

The impact of the design parameters on the floating node voltage accuracy as described in the literature provides guidance for our choices of device dimensions [see Fig. 1(a)]. ${ }^{27}$ We first review two mechanisms that lead to a random error in the floating node voltage and next describe two mechanisms that produce a systematic error. As we shall see, in general, a larger holding capacitance not only reduces these errors but also increases the footprint and power dissipation, both of which can limit scalability as well. ${ }^{12}$

Fundamentally, the voltage resolution $\Delta V$ of a floating node is limited by the electron charge, $e$, as

$$
\Delta V=e / C_{H},
$$

where $C_{H}$ is the total capacitance of the floating node to ground. It is dominated by the holding capacitor in our case. In order to keep $\Delta V$ below $1 \mu \mathrm{V}, C_{H}$ should be larger than $160 \mathrm{fF}$.

Next, thermal noise is present due to the transistor channel resistance when the transistor $\left(F E T_{1}\right)$ in a switched-capacitor circuit is switched on. The random thermal noise voltage is maintained on the holding capacitor after switching off the transistor. The root mean square (RMS) noise voltage on the capacitor is calculated as ${ }^{28}$

(a)

(c)

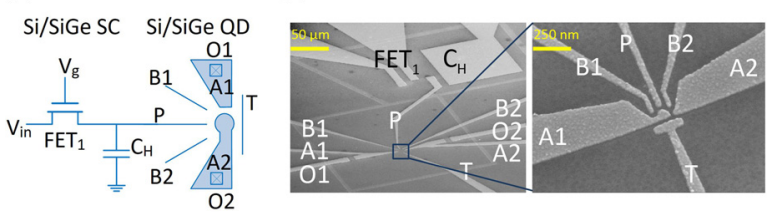

(b)

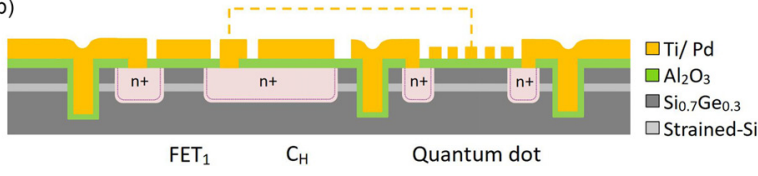

FIG. 1. (a) Device schematic showing the switched-capacitor circuit connected to gate $P$ of a single quantum dot. (b) Cross section of the device. All elements, the transistors, capacitors, and quantum dots are integrated on the same Si/SiGe based substrate and share the same aluminum oxide layer as dielectric. (c) SEM image of device $C$. The relevant device dimensions are listed in Table I.

$$
V_{n}^{r m s}=\sqrt{k_{B} T / C_{H}}
$$

For instance, to obtain a noise level below $1 \mu \mathrm{V}$ at a temperature of $10 \mathrm{mK}$, the holding capacitance must exceed $138 \mathrm{fF}$.

A first systematic offset in the floating node voltage is caused by channel charge injection. This effect refers to the charges that get redistributed to the drain and source upon switching a transistor off. ${ }^{29,30}$ Under the assumption that charges split equally to the source and drain, the error $\Delta V_{c}$ in the stored voltage on the floating node can be expressed as

$$
\Delta V_{c}=\frac{C_{\text {channel }}\left(V_{g}^{O N}-V_{\text {in }}-V_{t h}\right)}{2 C_{H}},
$$

with $C_{\text {channel }}$ being the capacitance between the transistor gate and channel, $V_{g}^{O N}$ the "on" voltage on the gate of $F E T_{1}, V_{\text {in }}$ the input voltage indicated in Fig. 1(a), and $V_{t h}$ the threshold voltage, which is the voltage difference between gate and source/drain at which charges begin to accumulate in the channel. For instance, when $V_{g}^{O N}$ is set $0.1 \mathrm{~V}$ higher than $V_{\text {in }}+V_{\text {th }}$, the holding capacitance needs to be 50 times larger than the transistor channel capacitance to keep $\Delta V_{c}$ below $1 \mathrm{mV}$.

Another factor that introduces systematic offsets in the maintained voltage is the parasitic capacitance from the transistor gate to the floating node. In series with the holding capacitance, it shifts the voltage on the floating node by an amount that depends on the voltage on the gate of $F E T_{1}$, given by ${ }^{29,30}$

$$
\Delta V_{p}=\Delta V_{g} \frac{C_{g s}}{C_{g s}+C_{H}},
$$

where $C_{g s}$ is the gate-source capacitance of $F E T_{1}$ and $\Delta V_{g}$ is the switching range used to turn the transistor on and off $\left(\Delta V_{g}=V_{g}^{\mathrm{ON}}-V_{g}^{\mathrm{OFF}}\right)$. Taking $\Delta V_{g}$ as $1 \mathrm{~V}$, the ratio of $C_{H}$ to $C_{g s}$ should exceed 1000 to keep $\Delta V_{p}$ below $1 \mathrm{mV}$.

Importantly, different from the random variations in the floating gate voltage, the systematic shifts can be accounted for in the calibration phase; hence, they do not impose strict requirements on $C_{H}$.

Turning now to power dissipation, the heat generated from the on-resistance of $F E T_{1}$ and the parasitic resistance on the leakage path can be expressed as

$$
P_{1}=C_{H} f_{g}\left(V_{1}-V_{2}\right)^{2},
$$

where $V_{1}$ and $V_{2}$ are the high and low voltages on the holding capacitor during operation and $f_{g}$ is the switching frequency of the transistor. Note that $P_{1}$ is proportional to the holding capacitance. If we refresh the floating node to compensate a $1 \mathrm{mV}$ drop with a $1 \mathrm{~Hz}$ frequency, the power dissipation of a single cell is $10^{-18} \mathrm{~W}$ when the holding capacitor is $1 \mathrm{pF}$. This is orders of magnitude smaller than the heat dissipated upon switching in the resistance in the line between the pulse generator and the transistor gate, which is given by

$$
P_{2}=\frac{1}{2} C_{\text {channel }} f_{g}\left(V_{g}^{O N}-V_{g}^{O F F}\right)^{2} .
$$

For a transistor with $0.01 \mathrm{pF}$ channel capacitance and $1 \mathrm{~V}$ switching range, the power dissipated on the signal line to its gate is $5 \times 10^{-13} \mathrm{~W}$. Even if we assume that this power is entirely dissipated on-chip, it would still allow $2 \times 10^{8}$ floating gate voltages to be 
maintained assuming $100 \mu \mathrm{W}$ available cooling power at the chosen operating temperature.

Making the transistors smaller reduces $C_{\text {channel }}$ and $C_{g s}$, which reduces switching power dissipation as well as the systematic shifts in the floating gate voltage. However, secondary effects appear when the device is scaled down. For instance, when the channel width is below $1 \mu \mathrm{m}$, the threshold voltage $V_{\text {th }}$ increases due to the narrow-channel effect. $^{31,32}$ Then, a higher gate voltage is required to turn on the transistor, which is more likely to cause hysteresis and breakdown.

Based on the above considerations, we made three devices with the same quantum dot design but different transistor and holding capacitor sizes and compared their voltage variations on the floating node both theoretically and experimentally. The device dimensions are listed in Table I (see also supplementary material, Appendix A). One practical consideration for the length of the transistor is the lateral diffusion of the implantation region, which is estimated to be $0.4 \mu \mathrm{m}$ in this process. The transistor channel length is chosen to be $10 \mu \mathrm{m}$ to reduce the effect of lateral diffusion on the actual length of the channel, though fundamentally the length could be much reduced.

For all devices, the quantum dot, transistor, and capacitor were integrated on a $\mathrm{Si}_{0.7} \mathrm{Ge}_{0.3} /$ strained-Si/buffered-SiGe heterostructure substrate. ${ }^{33}$ For the quantum dot, we used a single patterned metal layer to define the potential landscape that confines electrons. The top plate of the capacitor is formed by a metal gate and the bottom plate by a heavily implanted region in the semiconductor, with a dielectric separating the plates. The transistor is a field-effect transistor with the buried quantum well acting as the channel [see Fig. 1(b)].

The fabrication process began with the definition of markers followed by phosphorus ion implantation to create reservoirs for the quantum dots, the source and drain of the transistors, and the bottom electrode for the capacitors. This was followed by rapid thermal annealing at $700{ }^{\circ} \mathrm{C}$ to activate the dopants. Trenches of $100 \mathrm{~nm}$ deep and $10 \mu \mathrm{m}$ wide were subsequently etched into the $\mathrm{Si} / \mathrm{SiGe}$ substrate to isolate the devices from each other. A $20 \mathrm{~nm} \mathrm{Al}_{2} \mathrm{O}_{3}$ layer was then grown via atomic layer deposition to form the gate oxide for both quantum dots and transistors. Finally, we used electron beam lithography and lift-off to pattern an electron-beam evaporated 5/15 nm Ti/Pd stack to define the quantum dot gates, followed by patterning of a $5 / 195 \mathrm{~nm} \mathrm{Ti} / \mathrm{Pd}$ film for the transistor gate, the top electrode of the capacitor, and the leads and pads of the quantum dot. Figure 1(c)

TABLE I. Device dimensions and voltage variations on the floating node between static and floating mode tests.

\begin{tabular}{lccc}
\hline \hline & Device $A$ & Device $B$ & Device $C$ \\
\hline$C_{H}$ size $(\mu \mathrm{m} \times \mu \mathrm{m})$ & $15 \times 15$ & $15 \times 15$ & $100 \times 100$ \\
$C_{H}(\mathrm{pF})$ & 0.697 & 0.697 & 30.98 \\
$F E T_{1}$ size $(\mu \mathrm{m} \times \mu \mathrm{m})$ & $10 \times 10$ & $10 \times 1$ & $10 \times 1$ \\
$C_{\text {channel }}(\mathrm{pF})$ & 0.171 & 0.022 & 0.022 \\
$C_{g s}(\mathrm{fF})$ & 30 & 3.9 & 3.9 \\
Expected $\Delta V_{p}(\mathrm{mV})$ & 53.65 & 2.67 & 0.08 \\
Expected $\Delta V_{c}(\mathrm{mV})$ & $9.3-26.4$ & $2.9-3.5$ & $0.04-0.08$ \\
$\sqrt{k T / C_{H}}(\mu \mathrm{V})$ & 0.44 & 0.44 & 0.06 \\
$e / C_{H}(\mu \mathrm{V})$ & 0.23 & 0.23 & 0.0052 \\
Measured shift $(\mathrm{mV})$ & $44-48$ & $2.8-5.4$ & $0.5-1$ \\
\hline \hline
\end{tabular}

shows the SEM image of one of the final devices (Device $C$ in Table I). The details regarding the separate characterization of the single quantum dot and the transistor are described in supplementary material, Appendix B.

We mounted the device in a dilution refrigerator operating at a base temperature below $10 \mathrm{mK}$ and at zero magnetic field. All current measurements through the quantum dot were performed with a $100 \mu \mathrm{V}$ source-drain bias applied across the quantum dot, and all gate voltages were kept below the onset of hysteresis.

As a reference, the device was first tested in static mode with gate $P$ not floating (blue traces in Fig. 2). The current through the quantum dot was experimentally measured while the transistor was conducting. In the floating mode tests, we first turned on $F E T_{1}$ to charge the holding capacitor and then turned it off. After $10 \mathrm{~ms}$, we measured the current through the quantum dot while gate $P$ was floating. As shown in Fig. 2 (and Fig. S2 for device $A$ ), the patterns of the Coulomb peaks measured in floating mode were consistent with those measured in static mode but shifted in $V_{i n}$. Table I summarizes the expected and measured voltage shifts. The expected range of $\Delta V_{c}$ is calculated corresponding to the scan range of $V_{i n}$. The measured voltage shifts contain $\Delta V_{c}+\Delta V_{p}$. They are extracted from the shifts of the individual Coulomb peaks (at different $V_{i n}$ ), which thus also gives a range of voltage shifts. As can be seen from Table I, the measured shifts vs device dimensions match the predicted trend very well, with device $A$ showing the largest shifts and device $C$ the smallest shifts.

Nevertheless, there are still variations between the measured and expected voltage shifts. We here discuss this difference for device $B$. First, we note that the average voltage decay rate for device $B$ in the first $40 \mathrm{~s}$ after opening the transistor was approximately $2.8 \mu \mathrm{V} / \mathrm{s}$ (see supplementary material, Appendix C). This very low leakage rate compared to commercial DRAM is possible owing to the low operating temperature. The voltage shift on the floating gate due to leakage through the holding capacitor is thus negligible during the $10 \mathrm{~ms}$ interval between the moment the transistor is opened and the time of measurement. We will therefore compare the measured voltage shifts to those expected based on Eqs. (1)-(4). Figure S7(b) in the supplementary material, Appendix F shows the measured voltage shifts for the consecutive Coulomb peaks for device $B$. The overall trend of peak shift vs $V_{\text {in }}$ matches well with Eq. (3), expressing charge injection from the channel, for the five Coulomb peaks at the highest $V_{\text {in }}$ (the leftmost peak is shifted more than expected). The additional overall systematic shift is
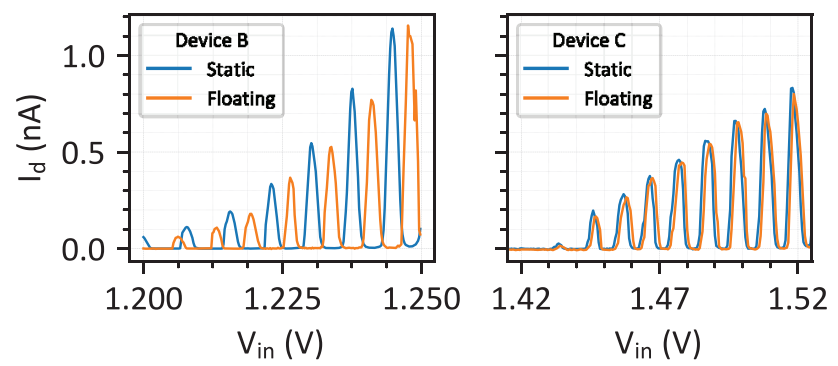

FIG. 2. Current through the quantum dot as a function of $V_{i n}$, with device $B$ (left panel) and $C$ (right panel) operated in static and floating mode. Patterns of Coulomb peaks are consistent between the blue (static mode) and orange (floating mode) traces. The voltage shift for device $C$ is less than that for device $B$ due to its larger holding capacitance. 
smaller than that expected based on Eq. (4) (the transistor gate voltage coupling in through the parasitic gate-source capacitance), possibly in part due to deviations in the estimated dielectric thickness or constant. In addition, the individual shifts do fluctuate around the overall linear trend by about $\pm 0.2 \mathrm{mV}$ (see supplementary material, Appendix E). By comparison, the random shifts expected from charge quantization and thermal noise [Eqs. (1) and (2)] are below $1 \mu \mathrm{V}$. However, the measured voltage fluctuations match well with the measured $1 / f$ noise caused by background charge fluctuations modulating the dot potential. ${ }^{34}$ The Coulomb peak measurement took a few minutes, and the $1 / f$ noise amplitude at $0.01 \mathrm{~Hz}$ is indeed of order $0.2 \mathrm{mV} / \sqrt{\mathrm{Hz}}$ (see supplementary material, Appendix D).

For qubit operation and readout, gate voltage pulses must be applied to one or more of the quantum dot gates. We now test the compatibility of applying such pulses with a switched-capacitor circuit present and operated in floating mode. In principle, the voltage pulses can be applied either to a floating gate (e.g., via the holding capacitor) or to another gate. Either way, the question is to what extent the presence of the capacitor and transistor that form the SC circuit distorts the waveform.

Here, we perform a preliminary test for voltage pulses applied to a gate that is not floating. Limited by the $1 \mathrm{kHz}$ sampling rate of the current measurement, we provided a $100 \mathrm{~Hz}$ square wave to gate $T$ [indicated in Fig. 1(a)] of device $C$ and checked whether the electrochemical potential of the quantum dot is able to follow the signal while gate $P$ is floating. The sequence of the experiment is depicted in Fig. 3. The input voltage $V_{\text {in }}$ is stepped through a range that covers several Coulomb peaks. For each $V_{i n}$, we first floated gate $P$ by setting $V_{g}$ from "high" to "low." Then, a $100 \mathrm{~Hz}, \pm 10 \mathrm{mV}$ pulse signal was applied to gate $T$ through a bias tee during $100 \mathrm{~ms}$, adding to a $790 \mathrm{mV}$ DC bias voltage, while we continuously measured the current flowing through the quantum dot. The current through the dot corresponding to the two stages of the voltage pulse, as well as the current during a subsequent time interval without gate voltages pulses, was extracted separately and compared to the static mode measurement results as shown in Fig. 3. The Coulomb peak patterns were consistent with the respective reference measurements. The $0.6-1.0 \mathrm{mV}$ voltage shift of the center peaks (blue solid vs dotted traces) is in agreement with the expected shift from channel charge injection and parasitic capacitance of the transistor upon switching off. Furthermore, the peaks obtained while applying a $100 \mathrm{~Hz}$ square pulse overlap closely with their expected positions, see the green and orange solid and dotted lines. The $0.6 \mathrm{mV}$ larger average shift for the orange vs the green solid lines indicates that the square pulse amplitude at the gate is slightly larger than the intended $\pm 10 \mathrm{mV}$, which is explained by a deviation (within the specified tolerance) of values of the attenuators placed in the transmission line connected to gate $T$. These results show that the voltage pulses on gate $T$ were not affected by the switched-capacitor circuit and by floating gate $P$. Based on electric circuit simulations (see supplementary material, Appendix G) that include the various capacitors discussed in the text, we expect the large capacitor that stores the floating node voltage not to impact the modulation of the dot potential in response to a pulse on gate $T$ until at least $20 \mathrm{GHz}$.

In summary, in this study, we demonstrated that a switchedcapacitor circuit placed between a quantum dot and demultiplexer can function as a local voltage source. The effects of channel charge injection and gate-source capacitive coupling introduce a systematic offset

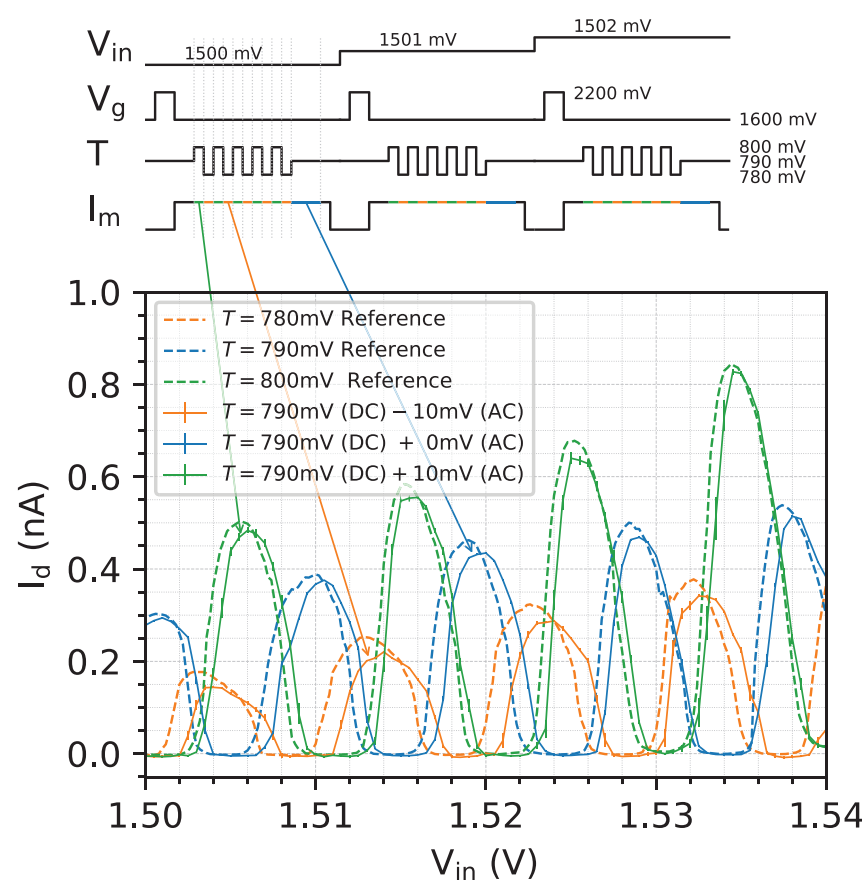

FIG. 3. Gate $P$ of device $C$ was floated for $200 \mathrm{~ms}$ for each value of $V_{\text {in. }}$ A 140 $\mathrm{mV}_{p p}, 100 \mathrm{~Hz}$ square wave was applied through a $23 \mathrm{~dB}$ attenuator to gate $T$ in the first $100 \mathrm{~ms}$, adding to a DC bias voltage of $790 \mathrm{mV}$ on the same gate. The current through the quantum dot was measured throughout these operations. Data corresponding to the high and low level of the voltage pulse $(T=800 \mathrm{mV}$ and $T=780$ $\mathrm{mV})$, and to the second $100 \mathrm{~ms}$ without pulses $(T=790 \mathrm{mV})$, are plotted separately as a function of $V_{\text {in }}$ (solid lines, the error bars indicate the standard deviation of each data point). The Coulomb peaks correspond very well to the static reference measurements, obtained with $780 \mathrm{mV}, 790 \mathrm{mV}$, and $800 \mathrm{mV} \mathrm{DC}$ voltages directly applied on gate $T$ while gate $P$ was not floating (dashed lines).

on the sampled voltage, which can be reduced if desired by using a larger holding capacitor and a smaller transistor. In the present measurements, random offsets in the stored voltage are dominated by $1 / f$ noise in the dot potential. Finally, we show that floating a quantum dot gate does not impact the effect of (slow) voltage pulses applied to another quantum dot gate. The incorporation of switched-capacitor circuits in future large-scale quantum processors can be facilitated using highly compact deep-trench capacitors and sparsely placed quantum dots. ${ }^{18}$

See the supplementary material for the specifications of devices $A, B$, and $C$; the method to define transistor on- and off-voltages on the integrated devices; the discharging rate and noise level on the plunger gate of device $B$; analysis of the voltage shift due to gatesource capacitance and channel charge injection; and the simulation results of the dot potential modulation in response to voltage pulses on gate $T$.

We thank Mark Eriksson for useful discussions, Stephan Philips for the design of the PCB onto which the sample was mounted, and Francisco Carrasco for assistance with sample fabrication. We acknowledge financial support from Intel 
Corporation and the QuantERA ERA-NET Cofund in Quantum Technologies implemented within the European Union's Horizon 2020 Programme.

\section{DATA AVAILABILITY}

The data that support the findings of this study are available at https://dx.doi.org/10.4121/12845870.v1.

\section{REFERENCES}

${ }^{1}$ B. M. Maune, M. G. Borselli, B. Huang, T. D. Ladd, P. W. Deelman, K. S. Holabird, A. A. Kiselev, I. Alvarado-Rodriguez, R. S. Ross, A. E. Schmitz, M. Sokolich, C. A. Watson, M. F. Gyure, and A. T. Hunter, "Coherent singlettriplet oscillations in a silicon-based double quantum dot," Nature 481, 344-347 (2012).

${ }^{2}$ E. Kawakami, P. Scarlino, D. R. Ward, F. R. Braakman, D. E. Savage, M. G. Lagally, M. Friesen, S. N. Coppersmith, M. A. Eriksson, and L. M. K. Vandersypen, "Electrical control of a long-lived spin qubit in A Si/SiGe quantum dot," Nat. Nanotechnol. 9, 666-670 (2014).

${ }^{3}$ D. M. Zajac, A. J. Sigillito, M. Russ, F. Borjans, J. M. Taylor, G. Burkard, and J. R. Petta, "Resonantly driven CNOT gate for electron spins," Science 359, 439-442 (2018).

${ }^{4}$ T. F. Watson, S. G. J. Philips, E. Kawakami, D. R. Ward, P. Scarlino, M. Veldhorst, D. E. Savage, M. G. Lagally, M. Friesen, S. N. Coppersmith, M. A. Eriksson, and L. M. K. Vandersypen, "A programmable two-qubit quantum processor in silicon,” Nature 555, 633-637 (2018).

5. Yoneda, K. Takeda, T. Otsuka, T. Nakajima, M. R. Delbecq, G. Allison, T. Honda, T. Kodera, S. Oda, Y. Hoshi, N. Usami, K. M. Itoh, and S. Tarucha, “A quantum-dot spin qubit with coherence limited by charge noise and fidelity higher than 99.9\%," Nat. Nanotechnol. 13, 102-106 (2018).

${ }^{6}$ X. Xue, T. F. Watson, J. Helsen, D. R. Ward, D. E. Savage, M. G. Lagally, S. Coppersmith, M. A. Eriksson, S. Wehner, and L. M. K. Vandersypen, "Benchmarking gate fidelities in a Si/SiGe two-qubit device," Phys. Rev. X 9, 201011 (2019).

${ }^{7}$ R. Pillarisetty, N. Thomas, H. C. George, K. Singh, J. Roberts, L. Lampert, P. Amin, T. F. Watson, G. Zheng, J. Torres, M. Metz, R. Kotlyar, P. Keys, J. M. Boter, J. P. Dehollain, G. Droulers, G. Eenink, R. Li, L. Massa, D. Sabbagh, N. Samkharadze, C. Volk, B. P. Wuetz, A.-M. Zwerver, M. Veldhorst, G. Scappucci, L. M. K. Vandersypen, and J. S. Clarke, "Qubit device integration using advanced semiconductor manufacturing process technology," in IEEE International Electron Devices Meeting (IEDM) (2018).

${ }^{8}$ M. Eriksson, S. Coppersmith, and M. Lagally, "Semiconductor quantum dot qubits," MRS Bull. 38, 794-801 (2013).

${ }^{9}$ D. Loss and D. P. DiVincenzo, "Quantum computation with quantum dots," Phys. Rev. A 57, 120-126 (1998).

${ }^{10}$ J. M. Elzerman, R. Hanson, L. H. Willems van Beveren, L. M. K. Vandersypen, and L. P. Kouwenhoven, "Semiconductor few-electron quantum dots as spin qubits," in Quantum Computing in Solid State Systems, edited by B. Ruggiero, P. Delsing, C. Granata, Y. Pashkin, and P. Silvestrini (Springer, New York, 2006), pp. 298-305.

${ }^{1}$ A. G. Fowler, M. Mariantoni, J. M. Martinis, and A. N. Cleland, "Surface codes: Towards practical large-scale quantum computation,” Phys. Rev. A 86, 032324 (2012).

${ }^{12}$ D. P. Franke, J. S. Clarke, L. M. K. Vandersypen, and M. Veldhorst, "Rent's rule and extensibility in quantum computing," Microprocessors Microsystems 67, 1-7 (2019).

${ }^{13}$ B. Keeth, R. J. Baker, B. Johnson, and F. Lin, DRAM Circuit Design. Fundamental and High-Speed Topics (Wiley-IEEE Press, 2007).

${ }^{14}$ C. Hill, E. Peretz, S. Hile, M. House, M. Fuechsle, S. Rogge, M. Simmons, and L. Hollenberg, "A surface code quantum computer in silicon," Sci. Adv. 1, e1500707 (2015).

${ }^{15}$ L. M. K. Vandersypen, H. Bluhm, J. S. Clarke, A. S. Dzurak, R. Ishihara, A. Morello, D. J. Reilly, L. R. Schreiber, and M. Veldhorst, "Interfacing spin qubits in quantum dots and donors-hot, dense, and coherent,” npj Quantum Inf. 3, 34 (2017).

${ }^{16} \mathrm{M}$. Veldhorst, H. Eenink, C. Yang, and A. Dzurak, "Silicon cmos architecture for a spin-based quantum computer," Nat. Commun. 8, 1766 (2017).

${ }^{17}$ R. Li, L. Petit, D. P. Franke, J. P. Dehollain, J. Helsen, M. Steudtner, N. K. Thomas, Z. R. Yoscovits, K. J. Singh, S. Wehner, L. M. K. Vandersypen, J. S. Clarke, and M. Veldhorst, "A crossbar network for silicon quantum dot qubits," Sci. Adv. 4, eaar3960 (2018).

${ }^{18}$ J. M. Boter, J. P. Dehollain, G. van Dijk, T. Hensgens, R. Versluis, J. S. Clarke, M. Veldhorst, F. Sebastiano, and L. M. K. Vandersypen, "A sparse spin qubit array with integrated control electronics," in IEEE International Electron Devices Meeting (IEDM) (IEEE, New York, 2019), pp. 31.4.1-31.4.4.

${ }^{19}$ R. K. Puddy, L. W. Smith, H. Al-Taie, C. H. Chong, I. Farrer, J. P. Griffiths, D. A. Ritchie, M. J. Kelly, M. Pepper, and C. G. Smith, "Multiplexed chargelocking device for large arrays of quantum devices," Appl. Phys. Lett. 107, 143501 (2015).

${ }^{20}$ S. J. Pauka, K. Das, R. Kalra, A. Moini, Y. Yang, M. Trainer, A. Bousquet, C. Cantaloube, N. Dick, G. C. Gardner, M. J. Manfra, and D. J. Reilly, "A cryogenic interface for controlling many qubits," arXiv:1912.01299v1 (2019).

${ }^{21}$ J. M. Elzerman, R. Hanson, L. H. Willems van Beveren, B. Witkamp, L. M. K. Vandersypen, and L. P. Kouwenhoven, "Single-shot read-out of an individual electron spin in a quantum dot," Nature 430, 431-435 (2004).

${ }^{22}$ A. Morello, J. J. Pla, F. A. Zwanenburg, K. W. Chan, K. Y. Tan, H. Huebl, M. Möttönen, C. D. Nugroho, C. Yang, J. A. van Donkelaar, A. D. C. Alves, D. N. Jamieson, C. C. Escott, L. C. L. Hollenberg, R. G. Clark, and A. S. Dzurak, "Single-shot readout of an electron spin in silicon," Nature 467, 687-691 (2010).

${ }^{23}$ H. Al-Taie, L. W. Smith, B. Xu, P. See, J. P. Griffiths, H. E. Beere, G. A. C. Jones, D. A. Ritchie, M. J. Kelly, and C. G. Smith, "Cryogenic on-chip multiplexer for the study of quantum transport in 256 split-gate devices," Appl. Phys. Lett. 102, 243102 (2013).

${ }^{24}$ D. R. Ward, D. E. Savage, M. G. Lagally, S. N. Coppersmith, and M. A. Eriksson, "Integration of on-chip field-effect transistor switches with dopantless Si/SiGe quantum dots for high-throughput testing," Appl. Phys. Lett. 102, 213107 (2013).

${ }^{25}$ S. Schaal, S. Barraud, J. J. L. Morton, and M. F. Gonzalez-Zalba, "Conditional dispersive readout of a CMOS single-electron memory cell,” Phys. Rev. Appl. 9, 054016 (2018).

${ }^{26}$ S. Schaal, A. Rossi, V. N. Ciriano-Tejel, T.-Y. Yang, S. Barraud, J. J. L. Morton, and M. F. Gonzalez-Zalba, "A CMOS dynamic random access architecture for radio-frequency readout of quantum devices," Nat. Electron. 2, 236-242 (2019).

${ }^{27}$ B. J. Sheu and C. C. Hu, IEEE J. Solid-State Circuits 19, 519-525 (1984).

${ }^{28}$ R. W. Brodersen, P. R. Gray, and D. A. Hodges, "MOS switched-capacitor filters," Proc. IEEE 67, 61-75 (1979).

${ }^{29} \mathrm{~B}$. Razavi, Design of Analog CMOS Integrated Circuits (McGraw-Hill Education, 2001).

${ }^{30}$ D. A. Johns and K. Martin, Analog Integrated Circuit Design (John Wiley and Sons, 1997).

${ }^{31}$ D. A. Neamen, Semiconductor Physics and Devices: Basic Principles, 4th ed. (McGraw-Hill, 2012).

${ }^{32}$ T. M. Lu, J. K. Gamble, R. P. Muller, E. Nielsen, D. Bethke, G. A. Ten Eyck, T. Pluym, J. R. Wendt, J. Dominguez, M. P. Lilly, M. S. Carroll, and M. C. Wanke, "Fabrication of quantum dots in undoped $\mathrm{Si} / \mathrm{Si}_{0.8} \mathrm{Ge}_{0.2}$ heterostructures using a single metal-gate layer," Appl. Phys. Lett. 109, 093102 (2016).

${ }^{33}$ N. Samkharadze, G. Zheng, N. Kalhor, D. Brousse, A. Sammak, U. C. Mendes, A. Blais, G. Scappucci, and L. M. K. Vandersypen, "Strong spin-photon coupling in silicon," Science 359, 1123-1127 (2018).

${ }^{34}$ B. M. Freeman, J. S. Schoenfield, and H. Jiang, "Comparison of low frequency charge noise in identically patterned $\mathrm{Si} / \mathrm{SiO}_{2}$ and $\mathrm{Si} / \mathrm{SiGe}$ quantum dots," Appl. Phys. Lett. 108, 253108 (2016). 